\title{
Neuropsychiatric symptoms in patients with thymoma- associated and non-thymoma myasthenia gravis
}

C Freeman, ${ }^{1}$ MB ChB, FCPsych (SA), MMed (Psych); I Lewis, ${ }^{1}$ BSc, MB ChB, FCPsych (SA); J M Heckmann, ${ }^{2}$ MB BCh, FCPNeurol (SA), PhD

${ }^{1}$ Department of Psychiatry and Mental Health, Faculty of Health Sciences, University of Cape Town, South Africa

${ }^{2}$ Division of Neurology, Department of Medicine, Faculty of Health Sciences, University of Cape Town, South Africa

Corresponding author: C Freeman (frmcar002@myuct.ac.za)

Background. Around 10 - 15\% of patients with myasthenia gravis (MG) have a thymoma, and non-motor symptoms are more frequent in these patients. We hypothesised that neuropsychiatric symptoms would also be more frequent.

Methods. A cross-sectional study of 30 consecutive MG patients attending a clinic at Groote Schuur Hospital, Cape Town, South Africa, was done over a 6-month period in 2010. Each patient underwent a series of single-blinded neuropsychiatric assessments, including the 16-item, self-reported Flanagan Quality of Life (QOL) scale, the Beck Depression Inventory second version, the Young Mania Rating Scale, the Hamilton Anxiety Rating Scale and the Brief Psychiatric Rating Scale (BPRS).

Results. The frequency and nature of neuropsychiatric symptoms were similar between thymoma ( $n=9)$ and non-thymoma $(n=21)$ MG patients. Symptoms of moderate or severe depression and anxiety were present in around $30 \%$. The severity of depression symptoms correlated with MG severity. Prednisone dosing was not associated with neuropsychiatric symptoms or QOL scores. Those with longer duration of MG were more likely to have higher scores on the BPRS and anxiety scales. Those with younger-onset MG had higher BPRS scores and a tendency to suicidal behaviour.

Conclusion. Although no association with thyoma was found, this study shows that neuropsychiatric conditions may be underdiagnosed in patients with MG. Systematic depression screening should be done at outpatient clinics, particularly for those who developed symptoms at a young age, those with severe disease and those with a long duration of illness. 


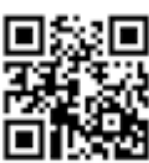

Myasthenia gravis (MG) is characterised by muscle fatigability, which may be chronic and debilitating. ${ }^{[1]}$ In patients who are positive for acetylcholine receptor antibodies, 10 - 15\% have a thymoma. Thymomas are removed surgically, but the associated MG usually persists independently. Patients with thymoma-associated MG often have more severe disease than non-thymoma-associated patients, and may have additional non-motor symptoms and frequently require longterm treatment. ${ }^{[2]}$ Psychiatric symptoms have been reported in MG patients, ${ }^{[3,4]}$ although little is known about their frequency or their relation to thymoma MG. The only published report documents three cases of psychosis in which psychotic symptoms persisted despite thymectomy ${ }^{[5]}$

We hypothesised that a patient with thymoma-associated MG will display a higher frequency of neuropsychiatric symptoms than patients with no thymoma. Here we report the findings of a pilot study in which both thymoma and non-thymoma MG patients were assessed using several neuropsychiatric questionnaires by investigators who were blind to thymoma status.

\section{Methods}

A cross-sectional study was done of 30 consecutive thymoma and nonthymoma MG patients attending a clinic at Groote Schuur Hospital, Cape Town, South Africa, over a 6-month period in 2010. Written informed consent was obtained and the University of Cape Town Health Sciences Human Research Ethics Committee approved the study.

\section{Study population}

All participants had MG and followed a standard treatment protocol that has been described elsewhere. ${ }^{[1]}$ Patients with thymoma MG were stabilised on immunosuppressive therapy and a thymectomy was done at the earliest possible opportunity. Thymectomies are offered to patients with moderately severe generalised seropositive MG $(<45$ years), even in the absence of thymoma.

\section{Interviews and tools}

Interviews were done on the day of patients' clinic visit by a psychiatrist, blinded to thymic status. Assessments included the following: 16-item self-reported Flanagan Quality of Life (QOL) scale, the Beck Depression Inventory second version (BDI-II), Young Mania Rating Scale (YMRS), the Hamilton Anxiety Rating Scale (HAM-A), and the Brief Psychiatric Rating Scale (BPRS) (Table 1). We defined suicidal behaviour as current suicidal ideation or one or more previous suicide attempts.

Demographic data and psychiatric history were also obtained during the interview. The quantified MG score, recent thyroid studies, comorbid conditions and current treatment were subsequently obtained by folder review. Age at symptom onset, age at diagnosis and thymic histology were obtained from an MG database. ${ }^{[1]}$ Participants were referred for further psychiatric management if indicated.

\section{Statistical analysis}

Data are presented as means and standard deviations (SDs), although skewed and ordinal data are presented as medians and interquartile ranges (IQRs). The Student $t$-test or Mann-Whitney $U$-test was used as appropriate and the Spearman rank-order correlation coefficient for ordinal data. Fisher's exact test was used for categorical data. A $p$-value (two-tailed) $<0.05$ was considered significant (Statistica 10 (Statsoft)).

\section{Results}

Of the participants, 21 (70\%) had non-thymoma MG and $9(30 \%)$ had thymoma MG. Age and gender distributions, duration of MG disease and symptoms before diagnosis, as well as distribution of MG symptoms and disease severity between the two groups, were similar. As expected, the quantified MG scores correlated with prednisone dosing ( $r s=0.39, p=0.034)$. Although all patients were receiving steroid-sparing agents, at least two-thirds of both groups were also on prednisone (76\%, non-thymoma MG; 66\%, thymoma MG, $p=0.76$ ). The average dose of prednisone was similar in the two groups (nonthymoma MG $16.3 \mathrm{mg} /$ day; thymoma MG $17.2 \mathrm{mg} /$ day, $p=0.86$ ). Eight of nine subjects with thymoma MG had already undergone thymectomy.

When neuropsychiatric symptoms across the non-thymoma and thymoma MG groups were compared, no statistical difference was seen in the frequency or severity of depression, anxiety or manic symptoms (Table 1). However, $33 \%$ of the total sample had moderate to severe symptoms of depression (thymoma $n=2$; non-thymoma $n=8$ ) and anxiety (thymoma $n=3$; non-thymoma $n=5$ ), which was associated with lower Flanagan QOL scores $(r s=-0.69, p=0.0001)$. Although symptoms of depression and anxiety did not always co-occur in the same patient, there was substantial overlap between the two as measured by the BDI-II and HAM-A scores $(r s=0.56, p=0.001)$. Higher MG severity scores were associated with higher BDI-II scores $(r s=0.51, p=0.004)$ and worse Flanagan QOL scores $(r s=-0.51, p=0.004)$, but not more severe anxiety symptoms $(r s=0.08, p=0.69)$.

Only four subjects with non-thymoma MG (amitriptyline $n=1$; fluoxetine $n=3$ ) and two with thymoma MG (amitriptyline $n=2$ ) were on antidepressant therapy at the time of the interview $(p=0.37)$. Patients with higher BDI-II scores were more likely to have been diagnosed with depression and started on antidepressant therapy; however, despite this intervention, these patients still had high scores on the BDI-II, which was associated with suicidal behaviour $(r s=0.68$, $p=\leq 0.0001$ ). Four subjects were newly identified with moderate to severe depression at the time of the interview (thymoma $n=1$; nonthymoma $n=3$ ).

In our sample, higher doses of prednisone were less frequent in those with symptoms of depression $(r s=0.28, p=0.14)$ or anxiety ( $r s=-0.08, p=0.66$ ). Interestingly, five patients met the criteria for hypomania, although these features were negatively associated with prednisone dose ( $r s=-0.38, p=0.034)$.

Overall, a longer duration of MG (based on symptom duration) was associated with both symptoms of anxiety $(r s=0.38, p=0.035)$ and depression ( $r s=0.37, p=0.045)$, but not with suicidal ideation $(r s=0.10$, $p=0.59$ ). A younger age at MG onset revealed a trend toward suicidal ideation or behaviour $(p=0.07)$; these subjects had higher scores on the multiple domain psychopathology measurement ( $r s=-0.48$, $p=0.007$ ), but neither higher depression or anxiety scores nor lower Flanagan QOL $(p=0.05)$. Taken together, almost half $(46 \%)$ of the participants interviewed required further psychiatric evaluation and management, in particular who developed MG symptoms at a young age. 
Table 1. Clinical and demographic characteristics of MG patients with or without thymoma, on the day of psychiatric interview

\begin{tabular}{|c|c|c|c|c|}
\hline & Total $(N=30)$ & Non-thymoma MG $(n=21)$ & Thymoma MG $(n=9)$ & $p$-value \\
\hline Male/female & $8 / 22$ & $5 / 16$ & $3 / 6$ & 0.61 \\
\hline Age, years, mean (SD) & $44.9(14.9)$ & $45.0(16.9)$ & $44.7(9.8)$ & 0.89 \\
\hline \multicolumn{5}{|l|}{ Age at MG symptom onset, years } \\
\hline Mean (SD) & $32.1(18.2)$ & $30.9(18.9)$ & $34.9(17.2)$ & 0.59 \\
\hline Median (IQR) & $32(16,44)$ & $25(16,43)$ & $39(29,44)$ & 0.50 \\
\hline Duration of MG since diagnosis, months, median (IQR) & $85(24,132)$ & $89(49,154)$ & $36(13,18)$ & 0.14 \\
\hline \multicolumn{4}{|l|}{ Marital status, $n(\%)$} & 0.23 \\
\hline Married/partner & $13(43)$ & $7(33)$ & $6(66)$ & \\
\hline Single & $10(33)$ & $8(38)$ & $2(22)$ & \\
\hline Divorced & $7(23)$ & $6(28)$ & $1(11)$ & \\
\hline Primary school & $4(13)$ & $2(9)$ & $2(22)$ & \\
\hline Secondary school & $14(47)$ & $12(57)$ & $2(22)$ & \\
\hline Tertiary study & $12(40)$ & $7(33)$ & $5(55)$ & \\
\hline Additional autoimmune disease, $n(\%)$ & $5(17)$ & $3(14)^{*}$ & $2(22)^{\dagger}$ & 0.62 \\
\hline \multicolumn{4}{|l|}{ MG symptoms, $n(\%)^{*}$} & \multirow[t]{5}{*}{0.47} \\
\hline Bulbar & $13(43)$ & $11(52)$ & $2(22)$ & \\
\hline Respiratory & $7(23)$ & $5(24)$ & $2(22)$ & \\
\hline Ocular & $16(53)$ & $12(57)$ & $4(44)$ & \\
\hline Limbs & $17(57)$ & $10(48)$ & $7(78)$ & \\
\hline QMGS, median (IQR)* & $9(4,11)$ & $8(5,11)$ & $10(4,11)$ & 0.76 \\
\hline Prednisone, $n(\%)$ & $22(73)$ & $16(76)$ & $6(66)$ & 0.61 \\
\hline Antidepressant therapy, $n(\%)$ & $6(20)$ & $4(19)$ & $2(22)$ & 0.37 \\
\hline Fluoxetine $20 \mathrm{mg}$ daily, $n(\%)$ & $3(10)$ & $3(10)$ & - & \\
\hline Amitriptyline 25 mg daily, $n$ (\%) & $3(10)$ & $1(3)$ & $2(7)$ & \\
\hline \multicolumn{5}{|l|}{ Neuropsychiatric assessments } \\
\hline BDI-II score, mean (SD) & $16(9)$ & $18(10)$ & $13(6)$ & 0.16 \\
\hline HAM-A score, mean (SD) & $14(9)$ & $14(9)$ & $13(9)$ & 0.67 \\
\hline BPRS score, mean (SD) & $12(7)$ & $13(7)$ & $10(6)$ & 0.30 \\
\hline YMRS score, mean (SD) & $4(4)$ & $4(4)$ & $4(4)$ & 0.87 \\
\hline Suicidal ideation, $n(\%)$ & $9(30)$ & $7(33)$ & $2(22)$ & 0.65 \\
\hline Psychiatric referral, $n(\%)$ & $14(46)$ & $11(52)$ & $3(33)$ & 0.42 \\
\hline \multicolumn{5}{|c|}{$\begin{array}{l}\text { MG = myasthenia gravis; } \mathrm{SD}=\text { standard deviation; } \mathrm{IQR}=\text { interquartile range; } \mathrm{QMGS}=\text { quantified myasthenia gravis score; } \mathrm{BDI}-\mathrm{II}=\text { Beck Depression Inventory }(\leq 13=\text { minimal; } 14-19=\text { mild; } \\
20-28=\text { moderate }) ; \mathrm{HAM}-\mathrm{A}=\text { Hamilton Anxiety Rating Scale }(14-17=\text { mild; } 18-24=\text { moderate); BPRS = Brief Psychiatric Rating Scale }(0-31=\text { mildly ill); YMRS = Young Mania Rating Scale } \\
(8-12=\text { hypomania). }\end{array}$} \\
\hline
\end{tabular}

\section{Discussion}

A cross-sectional neuropsychiatric evaluation of a sample of $30 \mathrm{MG}$ patients revealed prominent psychiatric symptoms. Although we did not find a difference in the frequency of neuropsychiatric symptoms between thymoma and non-thymoma MG patients as anticipated, one-third overall were found to be either clinically depressed or experiencing moderate to severe anxiety symptoms. In addition, one-third reported one or more past suicide attempts or exhibited suicidal ideation at the time of interview, and almost half of the sample required additional psychiatric evaluation and management, particularly those with a younger age of MG onset. Although 20\% were taking antidepressants, they reported ongoing symptoms of depression and/or anxiety. Three of these subjects were taking $25 \mathrm{mg}$ amitriptyline daily, a dose considered subtherapeutic for the 
treatment of depression and potentially contributing to ongoing symptoms. Medication adherence was not assessed in this study, but should be considered in cases of subclinical treatment response. A proportion of participants found to be depressed had not been previously diagnosed.

The high prevalence of psychiatric symptoms found in patients with MG, particularly depression and anxiety, correspond with previous reports. A similar-sized sample using a structured psychiatric interview (MINI-Plus) found $26 \%$ to have a depression disorder and $46 \%$ an anxiety disorder. ${ }^{[3]}$ We used the BDI-II to measure depression and found higher overall scores compared with two larger cohorts, North American and Japanese, who used the same tool. MG patients with high scores on the BDI-II were also at greater risk for suicidal behaviour. ${ }^{[4,6]}$ In addition, one-third of our sample reported moderate to severe anxiety symptoms. However, there was no correlation between anxiety and either thymoma status or prednisone dose. In contrast, a large Chinese cohort using the same scale, found $46 \%$ to have high anxiety scores correlating with both the presence of thymoma and prednisone dose. ${ }^{[7]}$

Our findings revealed a number of factors associated with a depressed state in MG: more severe disease, comorbid anxiety symptoms, suicidal ideation or behaviour, and concurrent antidepressant therapy. In contrast to a previous report, we found no association between the current prednisone dose and severity of mood or presence of anxiety symptoms. ${ }^{[4]}$ Although not apparent in our study, treatment with corticosteroids is well known to precipitate dose-dependent psychiatric disturbance, particularly affective disorders, and should be considered in the aetiology of psychopathology in MG.

We found five patients with hypomanic features on the YMRS, although the reliability of this scale is limited as it does not reflect the clinical picture over several days, which is needed to make the diagnosis of mania or hypomania. Mania and hypomania are often thought to arise as a consequence of corticosteroid therapy, but we found no correlation with prednisone dose. Only one of these patients was on antidepressants at the time of interview, and two had thymoma MG.

\section{Study limitations}

This study had several limitations. The sample size was small, increasing the possibility of a statistical type 2 error. Although the subjects were outpatients, those with more severe disease would be more likely to have frequent follow-up visits, introducing selection bias. Owing to limited resources, a battery of validated psychiatric rating scales was used in the structured psychiatric interview to assess depression, anxiety and manic symptoms based on those previously used in MG studies. A detailed psychiatric assessment examining broader psychopathology, e.g. cognitive impairment, personality disorders and substance abuse, was not undertaken. Although we enquired about social and occupational function, we omitted a specific tool assessing daily function in MG and did not assess socioeconomic status, which could affect psychopathology.

\section{Conclusion}

Neuropsychiatric symptoms did not differ between thymoma and non-thymoma MG patients in this sample. A high frequency of depression and anxiety was found in this MG cohort. Clinicians had overlooked psychiatric symptoms which, if untreated, would be likely to impact on the QOL and management of MG. Our results highlight the need for systematic depression screening in the outpatient clinic, particularly in patients who develop symptoms at a young age, those with severe disease and those with a long duration of illness.

Acknowledgements. The authors wish to thank the staff of the Division of Neurology, Groote Schuur Hospital, for their assistance with this study. Dr C P Freeman is a recipient of a Discovery Foundation Academic Award.

\section{References}

1. Heckmann JM, Owen EP, Little F. Myasthenia gravis in South Africans: Racial differences in clinical manifestations. Neuromuscul Disord 2007;17(11-12):929-934. [http://dx.doi. org/10.1016/j.nmd.2007.07.002

2. Suzuki S, Utsugisawa K, Suzuki N. Overlooked non-motor symptoms in myasthenia gravis. J Neurol Neurosurg Psychiatr 2013;84:989-994. [http://dx.doi.org/10.1136/jnnp-2012-304057]

3. Ybarra MI, Kummer A, Frota ERC, et al. Psychiatric disorders in myasthenia gravis. Arq Neurosiquiatr 2011;69(2A):176-179.

4. Suzuki Y, Utsugisawa K, Suzuki S, et al. Factors associated with depressive state in patient with myasthenia gravis: A multicentre cross-sectional study. BMJ Open 2011;1(2):e000313-3. [http://dx.doi.org/10.1136/bmjopen-2011-000313]

5. Musha M, Tanaka F, Ohuti M. Psychosis in three cases with myasthenia gravis and thymoma J Exp Med 1993;169(4):335-344.

6. Sitek EJ, Bilińska MM, Wieczorek D, Nyka WM. Neuropsychological assessment in myasthenia gravis. Neurol Sci 2009;30(1):9-14. [http://dx.doi.org/10.1007/s10072-008-0001-y]

7. Qui L, Feng $\mathrm{H}$, Huang X, et al. Study of incidence and correlation factors of depression, anxiety and insomnia in patients with myasthenia gravis. Zhonghua Yi Xue Za Zhi 2010;90(45):3176-3179. 